\title{
Page Kidney Secondary to Large Splenic Artery Aneurysm Bleeding and Its Management by Angioembolization
}

\author{
Santosh Kumar ${ }^{1, *}$; Kumar Jayant ${ }^{1}$; Sriharsha AS ${ }^{1}$; Sharwan Kumar Singh ${ }^{1}$; Swati Agrawal ${ }^{2}$ \\ ${ }^{1}$ Department of Urology, Postgraduate Institute of Medical Education and Research, Chandigarh, India \\ ${ }^{2}$ Department of Obstetrics and Gynecology, Medical College Udaipur, Rajasthan, India \\ ${ }^{*}$ Corresponding author: Santosh Kumar, Department of Urology, Postgraduate Institute of Medical Education and Research, Chandigarh, India. Tel: +91-9417374067, Fax: 91- \\ 1722744401, E-mail: santoshsp1967jaimatadi@yahoo.co.in
}

Received: December 24, 2013; Accepted: February 7, 2014

\begin{abstract}
Introduction: The Page kidney is a rare phenomenon that refers to hypertension resulting from any external compression of a kidney by a hematoma, tumor, lymphocele, or urinoma. The activation of the renin-angiotensin-aldosterone system is believed to be the main mechanism responsible for development of resistant hypertension in Page kidney.

Case Presentation: We reported a patient with chronic pancreatitis who presented with hypotension due to splenic artery aneurysmal bleed; following the resuscitation, accelerated hypertension secondary to Page kidney caused by perinephric hematoma presented. Early diagnosis by contrast-enhanced computed tomography of the abdomen and renal angiogram was followed by therapeutic angioembolization. However, ultrasound guided aspiration was not done because of denial by the patient for further treatment. Followup showed normalization of blood pressure and resolution of hematoma on subsequent abdomen ultrasound evaluation.

Discussion: Splenic artery aneurysm is a very uncommon cause of Page kidney and to our knowledge, it was the first case of its kind ever reported in the literature.
\end{abstract}

Keywords:Embolization, Therapeutic; Kidney; Spleen; Aneurysm

\section{Introduction}

The Page kidney is a rare condition that occurs due to any external compression of kidney by a hematoma, lymphocele, tumor, or urinoma leading to hypertension (HTN). This high-renin state HTN occurs due to activation of the renin-angiotensin-aldosterone system (RAAS) induced by renal hypoperfusion and ischemia (1). Most common causes attributed to the development of perinephric hematoma are traumatic or iatrogenic (e.g. renal biopsy, lumbar block, etc.). Other mentioned associations of Page kidney are renal tumor, renal cyst, vasculitis, and anticoagulation by coumarin. The interval between initial insult and the development of HTN can be as short as few days to as long as few years and may even present with hypertensive emergencies. We reported a case of hypotension due to the bleeding from splenic artery aneurysm followed by the accelerated HTN postresuscitation secondary to Page kidney. The underlying cause was a hematoma formed by aneurysmal bleeding in a patient with chronic pancreatitis who was treated by angioembolization. Patient was further followed on outpatient basis, which showed normalization of blood pressure. Subsequent ultrasound abdomen revealed resolution of hematoma.

\section{Case Presentation}

A 40-year-old male with a medical history of alcoholic liver disease and chronic pancreatitis attended at our emergency department with acute onset of hypotension and severe abdominal pain in epigastrium that was radiating towards back and was associated with nausea and vomiting. He had no history of HTN. On evaluation, he had anemia ( $\mathrm{Hb}, 7 \mathrm{mg} / \mathrm{dL}$ ), with normal findings on renal function tests (serum creatinine, $0.9 \mathrm{mg} / \mathrm{dL}$; urea, $21 \mathrm{mg} /$ $\mathrm{dL}$ ). Serum electrolyte assessment showed persistent hypokalemia (serum $\mathrm{K}^{+}$, 2.6-3.4 $\mathrm{mmol} / \mathrm{L}$ ) while serum magnesium was normal (serum $\mathrm{Mg}^{2+}, 1.45 \mathrm{mmol} / \mathrm{L}$ ). Ultrasonography of the whole abdomen showed left perinephric hematoma with normal-sized left kidney $(11 \mathrm{~cm})$; contralateral kidney was also normal. Subsequently, patient underwent contrast-enhanced computed tomography (CECT) of the abdomen with computed tomography angiography which showed large perinephric hematoma measuring $16.8 \times 14.7 \mathrm{~cm}$, secondary to splenic artery aneurysmal bleeding (Figure 1). There were no findings suggestive of renal artery stenosis on color Doppler ultrasound assessment. Plasma renin activity was $60 \mathrm{ng} /$ $\mathrm{mL} / \mathrm{h}$ (normal range, $0.2-2.8 \mathrm{ng} / \mathrm{mL} / \mathrm{h}$ ) with a plasma aldosterone level of $46.3 \mathrm{ng} / \mathrm{dL}$.

Implication for health policy/practice/research/medical education:

Page kidney is a rare cause of hypertension secondary to renin-angiotensin-aldosterone system activation. Although splenic artery aneurysm due to chronic pancreatitis is a very uncommon cause of Page kidney, early evaluation by contrast-enhanced computed tomography of the abdomen and renal angiogram is diagnostic and less invasive procedures such as angioembolization and percutaneous drainage promise good outcome.

Copyright (c) 2014, Nephrology and Urology Research Center; Published by Kowsar Corp. This is an open-access article distributed under the terms of the Creative Commons Attribution License, which permits unrestricted use, distribution, and reproduction in any medium, provided the original work is properly cited. 
After early assessment of patient's general condition, he was immediately resuscitated with intravenous fluids and three units of packed red blood cell transfusion, along with correction of hypokalemia under ECG monitoring. After initial resuscitation and stabilization, he was taken for angioembolization with coils that led to resolution of aneurysm (Figures 2 and 3). Postprocedure, patient was kept under monitoring. His vital signs remained stable with the hemoglobin level of $11 \mathrm{mg} / \mathrm{dL}$. The next day he developed accelerated HTN. Patient required a combination of antihypertensive drugs for its treatment. The patient refused any further surgical or percutaneous intervention although he was informed regarding the disease process and its consequences. He was discharged on medication.

\section{Discussion}

Page kidney was initially described by Page in 1939 (2). The main mechanism responsible for emergence of accelerated HTN and hypokalemia is believed to be RAASmediated. Although Page kidney seems to be similar to the Goldblatt model of renovascular HTN, the latter is due to compression or stenosis of major renal vessels. On the other hand, Page kidney seems to be the result of an ischemic renal vascular insult. Up to this date, approximately 110 cases are reported in literature (3). Most cases are due to compression of the kidney by a subcapsular or perinephric hematoma. Earlier blunt abdominal trauma was the major cause of this hematoma although nowadays many cases (ten out of 28 cases) are iatrogenic injuries following renal transplant biopsy of the kidney (4). In our case, the reason behind hypotension was bleeding from splenic artery aneurysm, which evolved as a complication to chronic pancreatitis, while the development of accelerated HTN was due to the perinephric hematoma. However, the case we reported here is the first of its kind. Up to this date, no case of Page kidney consequent to splenic artery aneurysm has been reported. Pathological Page kidney is seen in acute and chronic types. The acute type is due to the collection of blood or fluid in the subcapsular or perinephric space, which is often short lived, and the chronic type has a delayed onset and is caused by a fibrocollagenous scar compressing the renal parenchyma. Renal insufficiency is not usually seen because of normal perfusion of contralateral kidney, which maintains normal renal function. With time, renal function may gradually decline due to progressive shrinkage of the affected kidney. The development of HTN and deterioration of the renal function probably occurs due to the compression-induced interstitial nephritis. However, in cases of renal allograft or solitary kidney, renal insufficiency may need urgent surgical intervention. Page kidney can be diagnosed by multiple imaging techniques with each modality having its own advantages and disadvantages. Ultrasound is easily available, cheap, and noninvasive; however, small perinephric hematomas can be missed due to its high operator dependence. By assessing the vascularity, color Doppler ultrasound can be helpful not only in diagnosing Page kidney but also in excluding renal artery stenosis. Abdominal CECT is noninvasive and has high sensitivity and specificity in diagnosis of very small hematomas; hence, it has become the modality of choice. Magnetic resonance angiography is helpful in evaluating the age of hematomas and patency of renal vessels. Other investigations such as intravenous urography (IVU), nuclear scan, and renal arteriography also play a role in diagnosing perinephric hematoma.

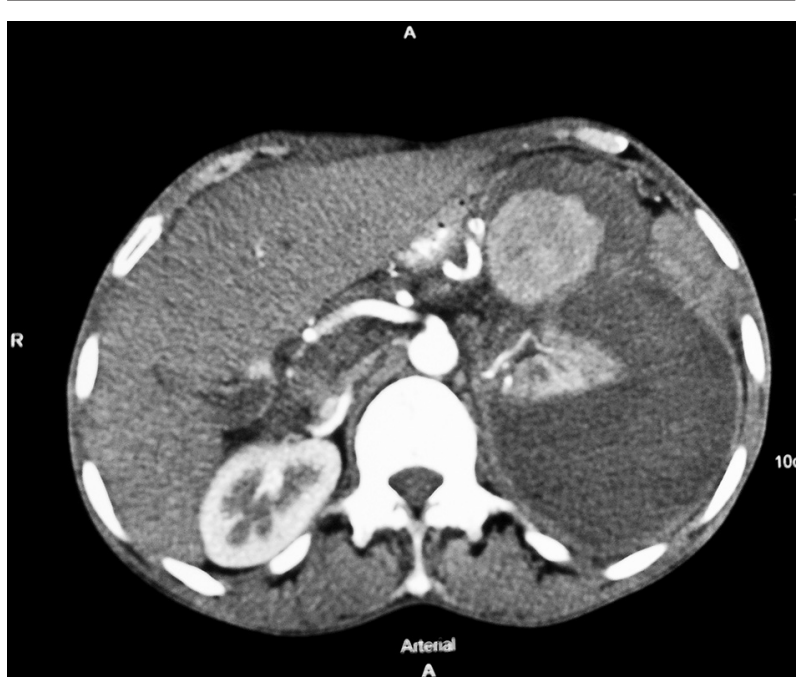

Figure 1. Contrast-Enhanced Computed Tomography of Abdomen Showing Large Splenic Aneurysm With Large Perinephric Hematoma Compressing the Kidney

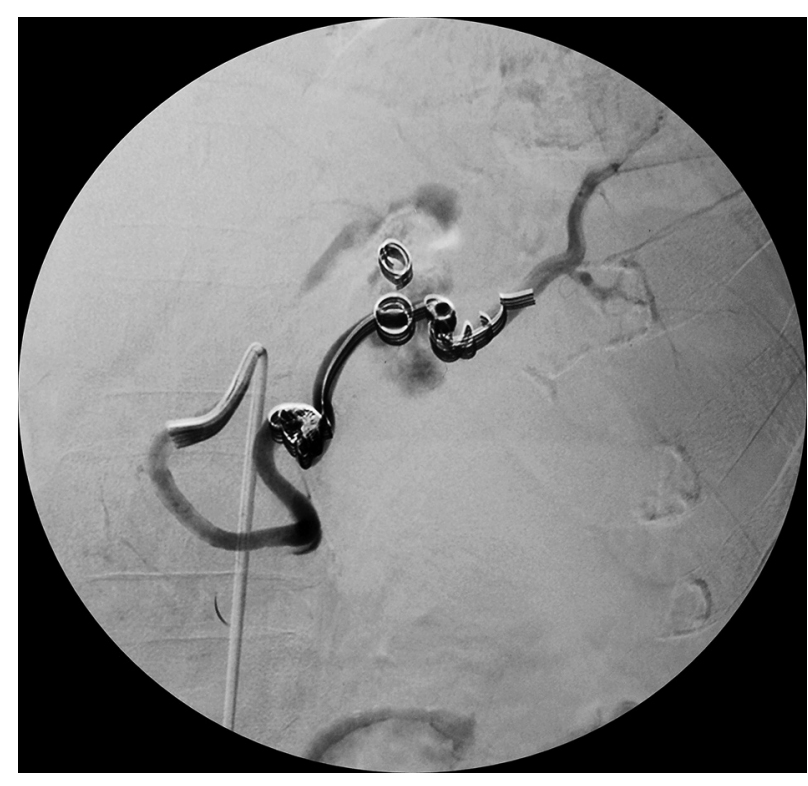

Figure 2. Digital Subtraction Angiography Depicting Angioembolization of the Splenic Artery Aneurysm 


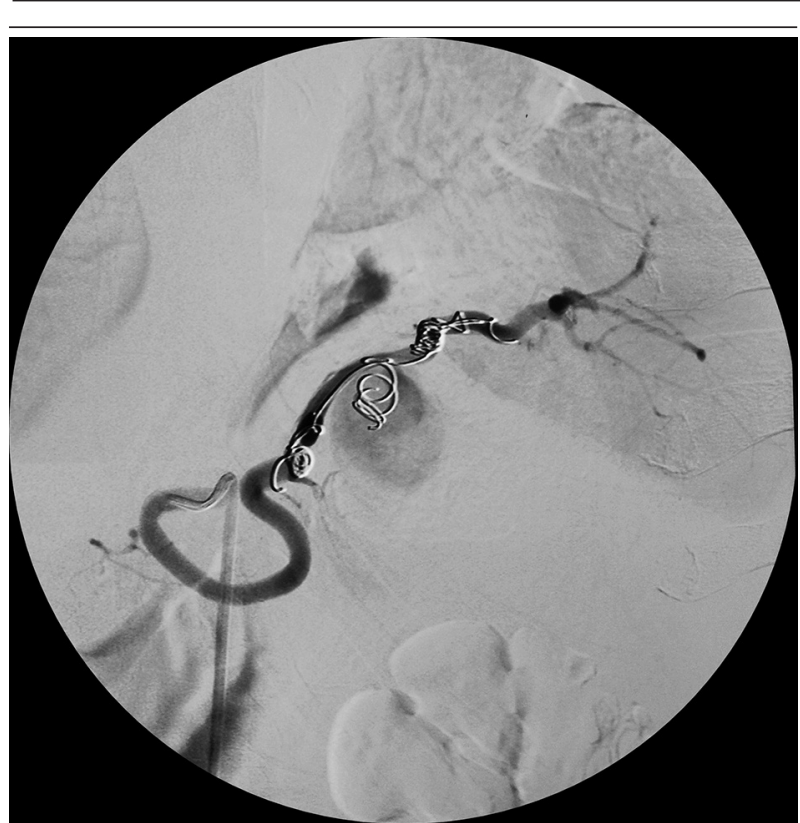

Figure 3. Digital Subtraction Angiography Picture Depicting Resolved Aneurysm After Embolization

The initial treatment usually involves drugs such as angiotensin convertase enzyme inhibitors (ACEIs) or aldosterone receptor blocker (ARB) with the aim of normalizing high blood pressure. The inability of a single agent to control the blood pressure necessitates the combination of multiple drugs such as beta blockers and calcium channel blockers (5). Earlier definitive treatment of Page kidney involved radical nephrectomy or open surgery to evacuate the hematoma or performing a decapsulating procedure to save the normal kidney $(6,7)$. The current approach is inclined toward less invasive procedures such as angioembolization and percutaneous drainage.

To summarize, Page kidney is a rare cause of HTN secondary to RAAS activation. Although splenic artery an- eurysm due to chronic pancreatitis is a very uncommon cause of Page kidney, early evaluation by CECT of abdomen and renal angiogram are diagnostic and less invasive procedures such as angioembolization and percutaneous drainage promise favorable outcome.

\section{Acknowledgements}

The authors express their gratitude to the patient for allowing them to publish the case report and use the images taken during his stay in hospital.

\section{Authors' Contribution}

All author contributed significantly in the manuscript.

\section{Financial Disclosure}

There was no financial interest.

\section{Funding/Support}

There was no funding source.

\section{References}

1. Elias AN, Anderson GJ, Dalakos TG, Streeten DH. Renin angiotensin involvement in transient hypertension after renal injury. $J$ Urol.1978;119(4):561-2.

2. IRVINE H, PAGE MD. The production of persistent arterial hypertension by cello- phane perinephritis .JAMA. 1939;113(23):2046-8.

3. Dopson SJ, Jayakumar S, Velez JC. Page kidney as a rare cause of hypertension: case report and review of the literature. Am J Kidney Dis. 2009;54(2):334-9.

4. Chung J, Caumartin Y, Warren J, Luke PP. Acute Page kidney following renal allograft biopsy: a complication requiring early recognition and treatment. Am J Transplant. 2008;8(6):1323-8.

5. Myrianthefs P, Aravosita P, Tokta R, Louizou L, Boutzouka E, Baltopoulos G. Resolution of Page kidney-related hypertension with medical therapy: a case report. Heart Lung. 2007;36(5):377-9.

6. McCune TR, Stone WJ, Breyer JA. Page kidney: case report and review of the literature. Am J Kidney Dis. 1991;18(5):593-9.

7. Jorge L, Alfredo P, Craig C. page kidney successfully treated with intrarenal artery embolization. Fed Pract. 2012;29(10):26-30. 